\title{
Removal of a high-fat diet, but not voluntary exercise, reverses obesity and diabetic-like symptoms in male C57BL/6J mice
}

\author{
Aikaterini Hatzidis, ${ }^{1 *}$ Jasmin A. Hicks, ${ }^{1,3 *}$ Rachel R. Gelineau, ${ }^{1}$ Nicole L. Arruda, ${ }^{1}$ \\ Isabella Monteiro De Pina, ${ }^{1}$ Karyn E. O’Connell, ${ }^{1,2}$ Joseph A. Seggio ${ }^{1}$ \\ ${ }^{1}$ Department of Biological Sciences, Bridgewater State University, Bridgewater, MA, USA; ${ }^{2}$ Takeda Pharmaceuticals, \\ Cambridge, MA, USA; ${ }^{3}$ Colorado State University, Molecular Cellular and Integrative Neuroscience Program, Fort Collins, \\ Colorado, USA \\ *Equal Contribution
}

\begin{abstract}
OBJECTIVE: Both the consumption of high-fat diets and exercise are known to produce alterations in metabolism and behavior. This study focuses on the effects of a change to a lowfat diet from a high-fat diet and voluntary exercise on obesity, type-2 diabetic-like symptoms, and locomotor behavior in male C57BL/6J mice. DESIGN: Mice were initially given either a high-fat diet or regular chow, along with a cage with a running-wheel to mimic exercise, or one without, to determine to what extend exercise affects these symptoms. Then half of the mice given a high-fat diet were switched to regular chow to ascertain if the switch in diet would improve type-2 diabetic-like and obesity symptoms. RESULTS: Wheel-running alone produced an improvement in insulin in mice continuously fed a high-fat diet $(p=0.006)$, but running-wheels did not produce any further improvements in mice with regular chow replacement $(p=0.999)$ or in controls $(p=0.996)$. Replacement of a high-fat diet with regular chow led to physiological improvements in insulin $(p=0.012)$ and leptin $(p<0.001)$, glucose tolerance $(p<0.001)$, and obesity $(p<0.001)$, more so than exercise alone. Mice consuming a high-fat diet without a wheel exhibited reduced home-cage activity compared to controls after the diet switch $(p=0.030)$, while no reduction was found in running-wheel activity between high-fat diet and regular chow consuming mice after switching diets $(p=0.516)$. CONCLUSIONS: These results suggest that exercise is only partially beneficial to improving health outcomes in mice consuming a high-fat diet, whereas incorporating a better diet, even without exercise, improves quality of health and can suppress T2DM symptoms and related conditions more so than exercise alone.
\end{abstract}

Key words: Exercise, High-fat diet, Locomotor, Mouse, Obesity, Type-2 diabetes, Wheel-running 


\section{INTRODUCTION}

The development of Type-2 Diabetes Mellitus (T2DM) is caused by a combination of genetic and environmental factors. Environmental factors leading to T2DM are consumption of high-fat diets and high-sugar diets along with a sedentary lifestyle, this typically contributing to the development of obesity that often, though not always, coincides with T2DM and increases the likelihood of developing insulin ${ }^{1}$ and leptin ${ }^{2}$ resistance. Mouse models are available for characterization of the progression of obesity and insulin and leptin resistance. Environmentally producing T2DM-like symptoms in rodent models is most commonly done through feeding a high-fat diet to susceptible strains (e.g., C57BL/6J (B6) mice), which, as a result develop insulin resistance, obesity, and hyperglycemia. ${ }^{3}$

The consensus is that moderate caloric restriction is the optimal way to lose weight and improve health. ${ }^{4}$ Human studies have shown that calorie restriction can produce improvements in insulin sensitivity even without increased physical activity. ${ }^{5}$ In animal models, reducing food consumption improves obesity, ${ }^{6}$ inflammation, and other metabolic processes. ${ }^{7}$ Another suggestion frequently offered by health professionals for decreasing the risk of developing obesity is increased activity and exercise. ${ }^{8}$ Earlier studies investigating the effects of exercise on weight loss have demonstrated that exercise can moderately reduce obesity in some cases. ${ }^{9}{ }^{10}$ But whether exercise can produce weight loss on its own has been called into question, as more recent studies have reported that exercise alone has no effect on weight. ${ }^{11,12}$ This latter observation appears to be corroborated by the fact that the United States has one of the highest levels of physical activity and yet one of the greatest rates of childhood obesity. ${ }^{13}$ Nevertheless, exercise, even without weight loss, is beneficial in alleviating many negative physiologic features of obesity/T2DM, including insulin resistance. ${ }^{14}$

In the rodent literature there are two methods commonly employed to mimic exercise: voluntary wheel-running where animals are given free access to a running-wheel, or forced exercise through treadmills or motorized running-wheels. Both voluntary wheel-running ${ }^{15,16}$ and forced exercise ${ }^{17,18}$ are effec- tive in improving health and reducing obesity in rodents fed a high-fat diet; however, these animals were still overweight and exhibited more unhealthy characteristics than sedentary animals on low-fat diets (low-fat diet). Switching high-fat diet fed rodents to a low-fat diet can promote weight loss, ${ }^{19}$ but there is some evidence showing that these animals may not fully recover from the obesogenic effects of the high-fat diet and remain heavier than their low-fat diet controls even after the high-fat diet is removed. ${ }^{20}$ However, few studies in the animal literature have investigated the combined effects of exercise and consumption of an improved diet on physiological characteristics or if the obesity and type-2 diabetic phenotype can be improved after a shorter timeframe of healthy behavior (weeks as opposed to months). Thus, the present study investigated whether the replacement of a high-fat diet with a healthier diet and the addition of exercise in the form of runningwheel access would promote more weight loss and improvements in T2DM-like symptoms than either exercise or calorie replacement alone in mice.

\section{METHODS}

\section{Ethical Statement}

All procedures performed in studies involving animals were approved by the Bridgewater State University Institutional Animal Care and Use Committee.

\section{Animals}

Sixty-six (66) male C57BL6/J (B6) mice were acquired from Jackson Laboratories (Bar Harbor, ME, USA) at six weeks of age, maintained in a 12:12 LightDark cycle, and given regular lab chow (physiological fuel value 3.36 kcals per gram; calorie percentages $13.4 \%$ fat, $29.8 \%$ protein, and $56.8 \%$ carbohydrate, Lab-Diet 5001, St. Louis, MO, USA) and water ad libitum. The mice were immediately housed individually with either running-wheel access (W) (wheel diameter: $23 \mathrm{~cm}$ ) or no running-wheel (NW) (IR-beam for home-cage activity recording, Star Life Sciences, Oakmont, PA, USA) from the start of the experiment. After one-week acclimation, the mice in each housing condition either continued with regular chow (RC) or were given a high-fat diet $(5.10 \mathrm{kcals}$ per gram, $61.6 \%$ fat, $18.1 \%$ protein, and $20.3 \%$ carbohydrate, TestDiet 58Y1). After nine additional weeks, the 
high-fat diet groups were further split into high-fat continuous (HFC - continuing to consume a high-fat diet) or high-fat replacement (HFR - RC replacing the high-fat diet) groups. Thus, this study employed six groups: HFC NW $(\mathrm{N}=10)$, HFC W $(\mathrm{N}=14)$, HFR NW ( $\mathrm{N}=10)$, HFR W $(\mathrm{N}=11), \mathrm{RC} N W(\mathrm{~N}=9), \mathrm{RC} W$ $(\mathrm{N}=12)$. Mice in the HFR groups (experiencing the food switch) were preselected prior to the start of the experimental treatments and were equally distributed to avoid any shelf effects and to avoid selecting only the most obese mice for the high-fat diet removal. In summary, all mice were subjected to one week of acclimation, nine weeks of treatment prior to food switch, and four weeks of post-food switch analysis. Weekly measurements of body mass and food and water intake were measured. All of the procedures described below were conducted during the middle of the light period. The timing of the tests described below is outlined in Figure 1.

\section{Activity Recording}

All mice had their wheel-running or home-cage locomotor activity recorded continuously throughout the experiment in their home-cage, as previously described. ${ }^{21}$ Wheel-running behavior was measured as number of wheel turns and home-cage activity was measured by number of infra-red beam breaks, both per 10 minute bin. Locomotor activity was analyzed as previously described. ${ }^{22}$

\section{Assessment of Glucose Tolerance}

Two Glucose Tolerance Tests (GTT) via intraperitoneal injection of $2 \mathrm{~g} / \mathrm{kg}$ glucose were conducted: one prior to HFR groups switch to RC (15 weeks of age) and a second after the food switch (Week 19). Twelve hours prior to testing, food was removed, wheels were locked, and new bedding was provided. Blood was collected via tail-prick and measured with One Touch Ultra 2 Glucose Monitors (Time 0), and then at 30,60 , and 120 minutes post-glucose injections.

\section{Measurement of Insulin, Leptin, and Triglyceride Levels}

Insulin, leptin, and triglyceride levels were measured prior to (Week 16) and after the food switch (Week 20), after a four-hour fast. Serum was obtained by centrifuging whole blood at $4{ }^{\circ} \mathrm{C}$ for 20 minutes at 2,000 $\mathrm{g}$ and hormone levels were measured using the Ultrasensitive Mouse Insulin and Leptin ELISA Kits (Crystal Chem, Downers Grove, IL, USA). Levels of

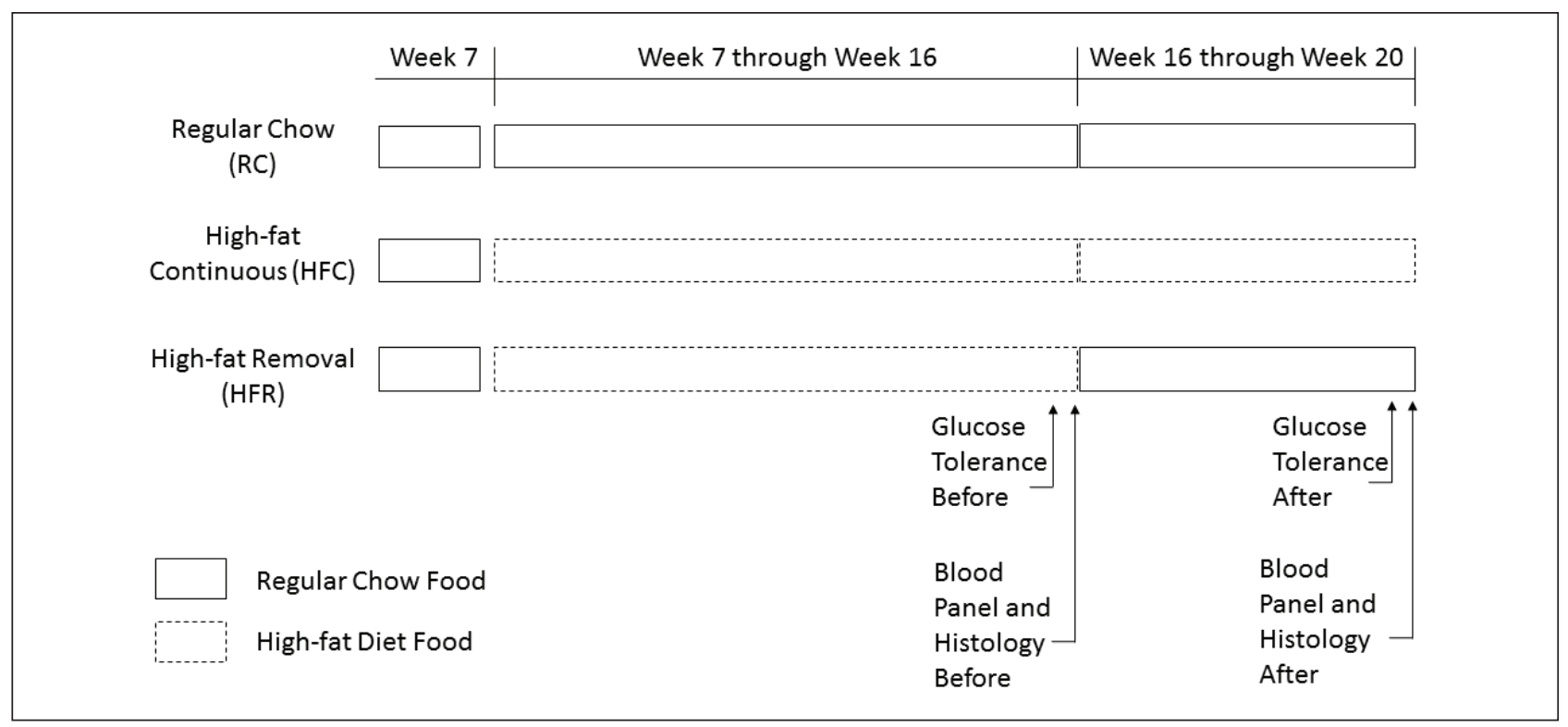

Figure 1. Experimental Protocols. The schematic of food availability and timing of physiological tests are outlined. Week 6 to 7 was the acclimation time where all groups received RC. From Week 7 to Week 16, HFC and HFR groups consumed a high-fat diet. From Week 17 to Week 20, HFR mice were given RC to replace the high-fat diet. Glucose tolerance tests were conducted during Week 15 and Week 19 for prior to and after the food switch, respectively. Blood and liver tissue were collected during Week 16 and Week 20 for prior to and after the food switch, respectively. NOTE: High-Fat Continuous/High-Fat Removal/High-Fat Diet Food. 
triglycerides were measured using the CardioChek System (Polymer Technology Systems Diagnostics, Indianapolis, IN, USA), by applying $15 \mu \mathrm{L}$ of whole blood onto the test strip.

\section{Histological Analysis}

This study used liver lipidosis as a measurement of obesity. Lipidosis of the liver was determined by identification of increased cytoplasmic vacuolation of hepatocytes with multiple clear discrete vacuoles, which indicates altered lipid metabolism and correlates with obesity in diabetics. ${ }^{23}$ Approximately one-third of each group were euthanized via $\mathrm{CO}_{2}$ narcosis 10 weeks into the experiment at the time of the food switch (16 weeks of age) to provide histological samples, while the rest continued in the experiment for an additional 4 weeks. Liver tissue was collected from each mouse and stored in phosphate-buffered $10 \%$-formalin solution for 3 weeks, then sent to the Dana-Farber/Harvard Cancer Center Rodent Histopathology Core (Boston, MA, USA) for processing and staining with hematoxylin and eosin (H\&E). Hepatic lipidosis was ranked for each animal using a scale of $0-3$, where 0 was normal, 1 was minimal, 2 was moderate, and 3 was severe.

\section{Statistical Analysis}

All results provided are represented as mean \pm SEM. Area under the curve (AUC) was calculated for each mouse for the GTT using Prism 5.0 (Graphpad Software, La Jolla, CA, USA). All statistical tests below were two-tailed, utilized $p<0.05$ as significance level, and were conducted using Systat 12.0 (Systat Software, Inc., San Jose, CA, USA). Paired t-tests with the Bonferroni correction for multiple comparisons were used to uncover before vs. after activity parameters. A Kruskal-Wallis test was used to analyze the rankings of hepatic lipidosis. Weight and kcals consumed were analyzed via a repeated measures two-way ANOVA and two-way ANOVAs were conducted to determine differences among the different groups, with Tukey pairwise comparisons, for all other dependent variables.

\section{RESULTS}

\section{Body Mass and Energy Intake}

Prior to high-fat diet access (Week 7) there were no differences in body mass among any of the groups for either diet ( $p=0.351)$ or wheel access $(p=0.168)$. For prior to the diet switch (Weeks 8 through 16), between groups repeated measures uncovered both a diet and wheel difference, but no interaction. The HFC and HFR groups (which were both consuming a high-fat diet) showed increased body weight over time compared to RC mice $(p<0.001)$. Additionally, running-wheel access also produced significantly reduced body weight compared to sedentary controls, regardless of diet $(p=0.012)$. At 16 weeks, HFR mice were given $\mathrm{RC}$ food to replace the high-fat diet. From Weeks 17 through 20 (after the switch), diet still produced significant alterations to body weight, whereby HFC mice were larger than HFR and RC $(p<0.001)$; however, the running-wheel was no longer modifying body mass $(p=0.375)$ (Figure $2 \mathrm{a})$. Moreover, total weight gain (Week 20 minus Week 7) was significantly different in mice consuming different diets regardless of running-wheel access. The mean difference $(\mathrm{g})$ for HFC compared with HFR and RC was 8.29 and $9.12 \mathrm{~g}$, respectively (both $p<0.001$ ), while the mean difference between RC and HFR was $0.83 \mathrm{~g}(p=0.846)$.

Prior to the switching of the diets, a two-way repeated measures ANOVA uncovered a diet and wheel difference concerning weekly kcals consumed among groups but no interaction. Both HFC $(p<0.001)$ and HFR $(p<0.001)$ consumed fewer kcals than RC prior to the food switch. Furthermore, animals with runningwheel access regardless of diet also consumed more kcals compared to mice without a wheel $(p<0.001)$. From Weeks 17 through 20, food intake was not different between diet groups $(p=0.579)$, but mice with running-wheels still consumed more kcals per week during this timeframe than sedentary animals $(p=0.036)$ (Figure 2b).

\section{Activity Patterns}

Home-cage and wheel-running activity are presented and summarized in Table 1. Prior to high-fat diet removal, there were no differences between the HFR and HFC groups as concerns total activity ( $p=0.949)$; both HFC ( $p=0.999)$ and HFR $(p=0.953)$ groups exhibited similar levels of locomotor activity both on and off running-wheels compared to RC. As expected, running-wheel access increased overall activity compared to home-cage activity $(p<0.001)$. 

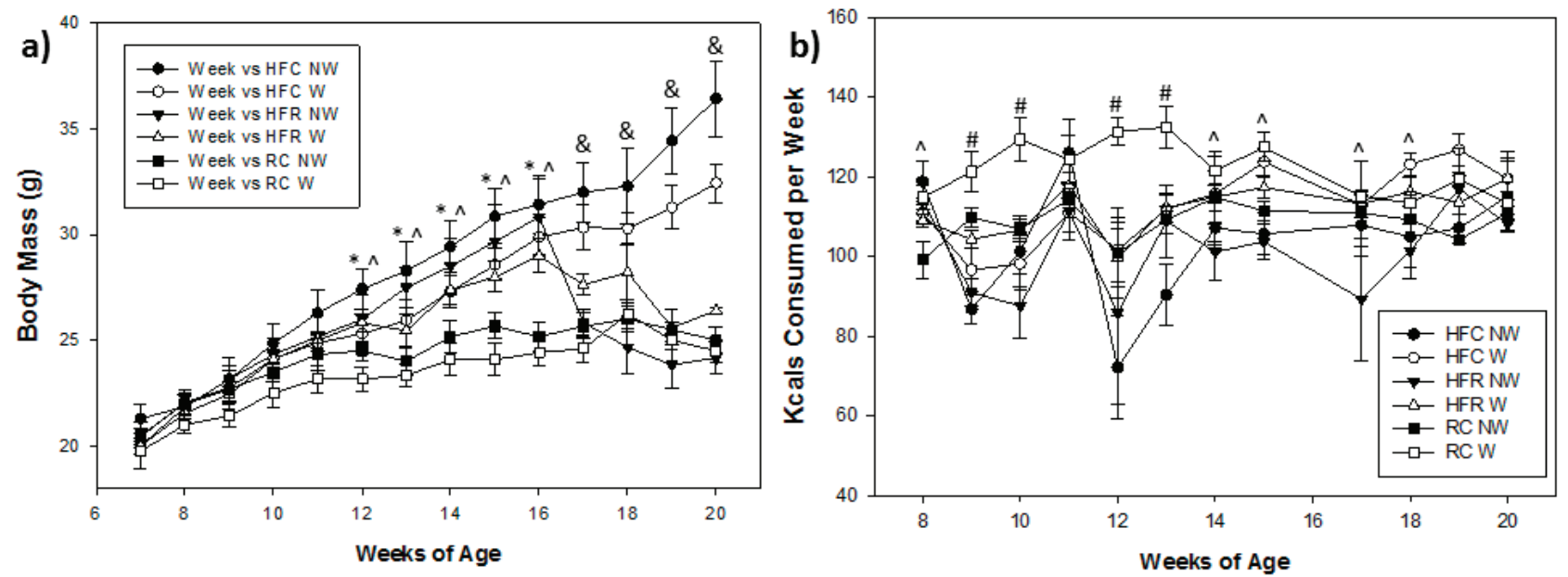

Figure 2. Body Mass, food consumption. a) Body Mass (grams). b) Energy Consumed (kcals per week). The dashed line indicates the time of the diet switch where prior to the switch is on the left and afterwards is to the right. * indicates HFC and HFR groups greater than RC groups, $\wedge$ indicates HFC groups greater than HFR and RC groups, $\ddagger$ indicates Wheel difference, and \# indicates RC greater than HFC and HFR, all at $p<0.05$.

Table 1. Activity Profile and bout analysis (mean \pm SEM) for all diet and running-wheel conditions. The top portion of the Table (Before) is activity exhibited prior to the food switch (Weeks 8-16), while the bottom part (After) is activity recorded after the RC replacement (Weeks 17-20). Italics indicate significant differences between Before and After activity parameters, at $p<0.05$.

\begin{tabular}{lccccccc}
\hline Before & & & & & & \\
\hline Diet & Wheel & Average & Light Average & Dark Average & Bout Length (min) & Counts per Bout & Bouts per Day \\
\hline RC & NW & $25.83 \pm 2.15$ & $12.48 \pm 1.53$ & $39.23 \pm 3.05$ & $42.70 \pm 2.20$ & $290.18 \pm 29.50$ & $11.35 \pm 1.90$ \\
RC & W & $52.09 \pm 4.15$ & $6.10 \pm 1.47$ & $98.08 \pm 8.14$ & $64.33 \pm 7.86$ & $1093.79 \pm 164.44$ & $7.20 \pm 0.80$ \\
HFC & NW & $17.60 \pm 1.13$ & $7.27 \pm 0.63$ & $27.91 \pm 1.80$ & 38.812 .05 & $209.61 \pm 19.23$ & $11.00 \pm 0.41$ \\
HFC & W & $60.30 \pm 5.40$ & $4.58 \pm 1.14$ & $115.99 \pm 9.99$ & $116.47 \pm 10.99$ & $2219.35 \pm 254.00$ & $4.43 \pm 0.28$ \\
HFR & NW & $19.21 \pm 1.62$ & $7.50 \pm 0.71$ & $32.73 \pm 2.70$ & $40.65 \pm 1.76$ & $241.53 \pm 20.13$ & $10.67 \pm 0.34$ \\
HFR & W & $55.85 \pm 3.99$ & $3.86 \pm 1.16$ & $107.99 \pm 7.65$ & $89.72 \pm 7.15$ & $1584.19 \pm 182.95$ & $5.17 \pm 0.30$ \\
\hline After & & & & & & & \\
\hline RC & NW & $25.23 \pm 2.54$ & $11.35 \pm 1.98$ & $39.15 \pm 3.70$ & $34.99 \pm 2.99$ & $226.10 \pm 32.55$ & $12.18 \pm 0.50$ \\
RC & W & $50.82 \pm 4.07$ & $5.98 \pm 1.50$ & $95.65 \pm 7.80$ & $56.11 \pm 6.88$ & $923.36 \pm 136.55$ & $7.90 \pm 0.74$ \\
HFC & NW & $11.97 \pm 0.55$ & $4.93 \pm 0.48$ & $19.04 \pm 0.79$ & $29.19 \pm 0.69$ & $127.56 \pm 6.46$ & $12.20 \pm 0.61$ \\
HFC & W & $60.59 \pm 5.05$ & $4.77 \pm 1.13$ & $108.41 \pm 12.00$ & $71.40 \pm 9.04$ & $1268.36 \pm 196.24$ & $5.14 \pm 0.42$ \\
HFR & NW & $21.30 \pm 2.93$ & $6.90 \pm 0.95$ & $35.69 \pm 5.02$ & $41.20 \pm 2.40$ & $265.47 \pm 28.38$ & $11.70 \pm 0.22$ \\
HFR & W & $45.31 \pm 3.00$ & $2.65 \pm 0.73$ & $87.96 \pm 5.70$ & $45.48 \pm 3.69$ & $661.65 \pm 88.32$ & $7.63 \pm 0.42$ \\
\hline
\end{tabular}

Mice consuming a high-fat diet had no issues running on the wheel. After switching diets, before vs. after analysis revealed that HFC NW mice exhibited reduced overall activity $(p=0.030)$, dark activity $(p=0.009)$, bout length ( $p=0.039$ ), and counts per bout ( $p=0.005$ ) as time progressed, but no change occurred in light activity ( $p=0.057$ ) or bouts per day ( $p=0.999$ ). This reduction in activity was not observed in the other sedentary mice: $\mathrm{RCNW}(p=0.195, p=0.930, p=0.292$, $p=0.170)$ or HFR NW ( $p=0.475, p=0.592, p=0.885$, $p=0.999$ ), nor in mice consuming a high-fat diet continuously with running-wheel access (HFC W) $(p=0.999, p=0.589, p=0.052, p=0.100)$ as regards overall activity, dark activity, bout length, and counts per bout, respectively. In summary, sedentary mice continuously consuming a high-fat diet exhibited 
reduced activity overall during their active phase, which was a result not observed in mice which did not consume a high-fat diet throughout the experiment or for mice given running-wheel access.

\section{Glucose Tolerance}

A GTT was conducted before switching the HFR groups to RC (Figure 3a, b). Mean fasting glucose levels $(\mathrm{mg} / \mathrm{dL})$ prior to the diet switch were as follows: HFC NW 219 \pm 20 , HFC W 191 \pm 9 , HFR NW 208 \pm 16 , HFR W 215 \pm 11 , RC NW 169 \pm 11 , RC W $140 \pm 6$. Fasting (Time 0 ) glucose was significantly increased in HFC $(p<0.001)$ and HFR $(p<0.001)$ compared to the $\mathrm{RC}$ groups, though they were similar to each other ( $p=0.908)$. Mean fasting glucose levels prior to the diet switch were as follows: HFC NW 50487 \pm 3623 , HFC W 44537 \pm 2298 , HFR NW 42917 \pm 2633 , HFR W
42538 \pm 2895 , RC NW 29115 \pm 4276, RC W 21909 \pm 580 . HFC $(p=0.001)$ and HFR $(p=0.001)$ groups exhibited slower glucose clearance than RC $(p=0.001)$; HFC and HFR mice exhibited similar glucose tolerance $(p=0.148)$. Wheel-running did not affect fasting glucose ( $p=0.143$ ) but improved glucose tolerance regardless of diet $(p=0.041)$.

The second GTT was performed three weeks after removing the high-fat diet and replacing it with $\mathrm{RC}$ for the HFR mice (19 weeks of age) (Figures 3c, d). Mean fasting glucose levels ( $\mathrm{mg} / \mathrm{dL})$ after the switch in diet were as follows: HFC NW 219 \pm 21 , HFC W $190 \pm 9$, HFR NW 125 \pm 9 , HFR W 138 \pm 8 , RC NW $150 \pm 9, \mathrm{RC} W 131 \pm 6$. HFC groups had elevated fasting glucose in contrast to HFR $(p<0.001)$ and RC groups $(p<0.001)$. AUCs after the switch in diet were as fol-
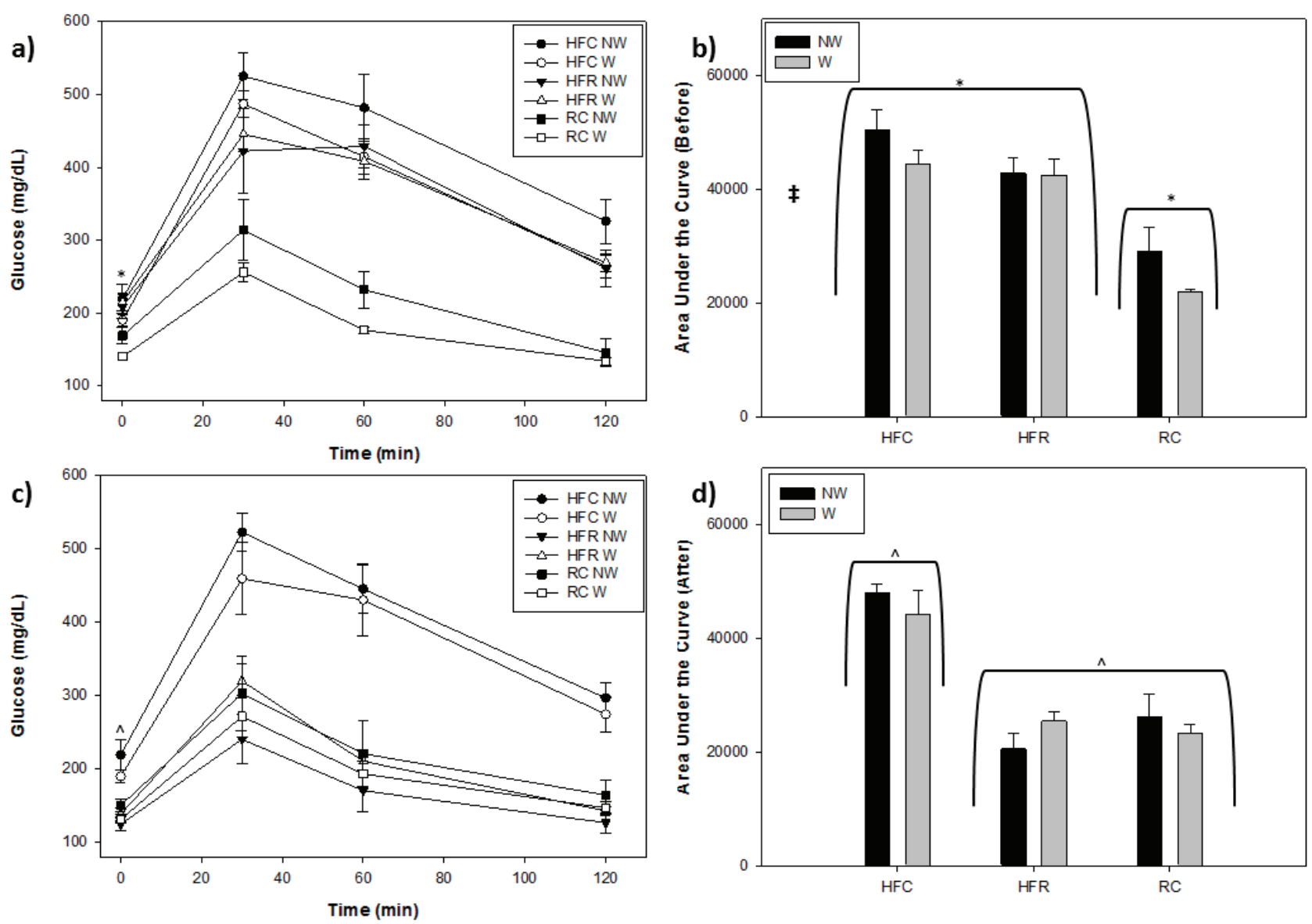

Figure 3. Glucose Tolerance Tests (GTT) with Area Under the Curve (AUC). a) Glucose levels over time of GTT for Before the diet switch. b) Area Under the Curve Before c) Glucose levels over time for After the diet switch. d) Area Under the Curve After the food switch. Time 0 was used to assay 12-h fasting glucose levels. * indicates HFC and HFR groups greater than RC groups, $\wedge$ indicates HFC groups greater than HFR and RC groups, and $\$$ indicates Wheel difference, all at $p<0.05$. 
lows: HFC NW 47931 \pm 1762 , HFC W 44258 \pm 4135 , HFR NW 20559 \pm 2668 , HFR W $25437 \pm 1579$, RC NW 26214 \pm 3935 , RC W 23205 \pm 1669 . HFC mice showed poorer glucose clearance compared to HFR $(p<0.001)$ and RC $(p<0.001)$; HFC and RC were similar to each other ( $p=0.947)$. Wheel-running did not improve glucose tolerance $(p=0.553)$ or fasting glucose $(p=0.199)$ among any group.

\section{Blood Panel}

Insulin, leptin, and triglycerides were measured before and after switching foods (Figures 4a, b, c, respectively). Mean insulin levels $(\mathrm{ng} / \mathrm{mL})$ prior to the diet switch were as follows: HFC NW 1.91 \pm 0.49 , HFC W 0.85 \pm 0.04 , HFR NW 2.11 \pm 0.36 , HFR W $0.99 \pm 0.43, \mathrm{RC} N W 0.76 \pm 0.08$, RC W $0.31 \pm 0.06$. Prior to $\mathrm{RC}$ replacement, insulin levels were significantly lower in $\mathrm{RC}$ groups than in HFC $(p=0.019)$ and HFR ( $p=0.003$ ), while HFC and HFR groups were not different $(p=0.834)$. Wheel-running, regardless of diet, produced improvements in insulin $(p=0.001)$. After switching the food source for HFR mice, the mean insulin levels were as follows: HFC NW $3.82 \pm 0.68$, HFC W 2.21 \pm 0.37 , HFR NW $1.30 \pm 0.67$, HFR W $0.58 \pm 0.06$, RC NW 0.52 \pm 0.08 , RC W $0.65 \pm 0.08$. Animals animals consuming HFD benefited from wheel access, but RC consuming animals did not ( $p=0.012$ ). More specifically, running-wheels were effective in reducing hyperinsulinemia in HFC mice ( $p=0.006$ ) compared to sedentary ones; however, they were ineffective in further reducing insulin levels for HFR ( $p=0.999)$ and RC ( $p=0.999)$. HFR and RC groups exhibited similar insulin levels $(p=0.980)$ while having reduced insulin compared to $\operatorname{HFC}(p<0.001)$.
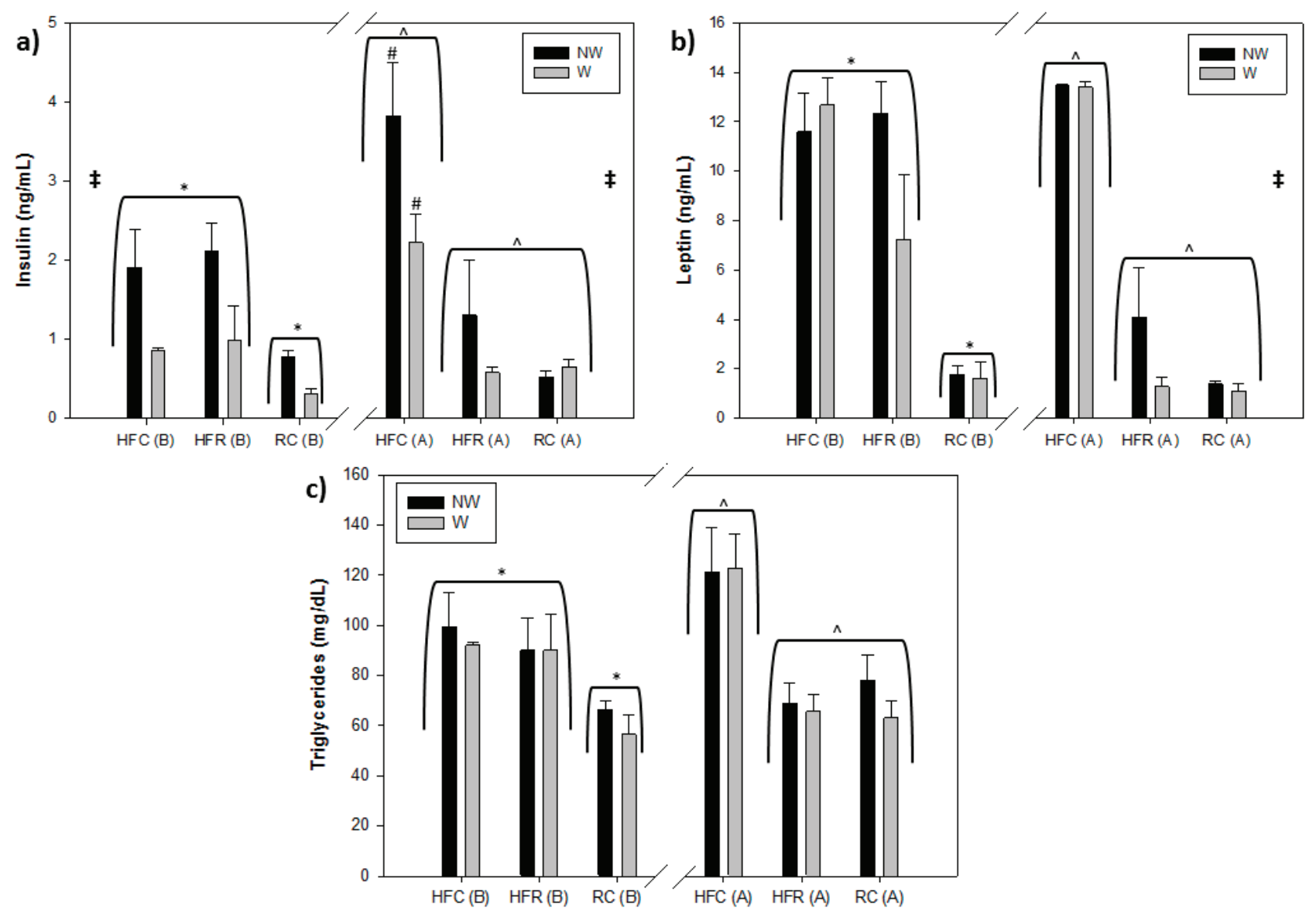

Figure 4. Blood panel for Before and After the food switch. a) Insulin Levels b) Leptin Levels c) Triglyceride Levels. * indicates HFC and HFR groups greater than RC groups, $\wedge$ indicates HFC groups greater than HFR and RC groups, \# indicates significant pairwise difference, $\ddagger$ indicates Wheel difference, all at $p<0.05$. The left side of the graph shows before (indicated by B) and the right side shows after food switch (indicated by A). 
Mean leptin levels (ng/mL) prior to the diet switch were as follows: HFC NW $11.59 \pm 1.56$, HFC W 12.66 \pm 1.10 , HFR NW 12.34 \pm 1.26 , HFR W 7.23 \pm 2.61, RC NW $1.79 \pm 0.36$, RC W $1.61 \pm 0.66$. Prior to RC replacement, leptin levels were significantly lower in RC groups than in HFC $(p=0.001)$ and HFR $(p=0.001)$, while HFC and HFR groups were not different ( $p=0.216$ ). Wheel-running, regardless of diet, produced no alterations in leptin levels ( $p=0.188$ ) during the first measurement. Mean leptin levels $(\mathrm{ng} / \mathrm{mL})$ after the diet switch were as follows: HFC NW 13.43 \pm 0.10 , HFC W 13.40 \pm 0.20 , HFR NW $4.10 \pm 1.97$, HFR W $1.28 \pm 0.39$, RC NW $1.37 \pm 0.13$, RC W 1.10 \pm 0.29 . After switching diets, HFC exhibited increased leptin levels compared to HFR $(p<0.001)$ and $\mathrm{RC}(p<0.001)$, while HFR and RC groups were similar to each other $(p=0.150)$. Regardless of diet, wheel-running produced reduced leptin levels ( $p$ $=0.050$ ) during the second time point.

Mean triglyceride levels (mg/dL) prior to the diet switch were as follows: HFC NW 99.5 \pm 13.4 , HFC W 92.0 \pm 1.4 , HFR NW 90.0 \pm 12.7 , HFR W $90.1 \pm 14.1$, RC NW 66.5 \pm 3.5 , RC W 56.5 \pm 7.8. Prior to $\mathrm{RC}$ replacement, triglyceride levels were significantly lower in RC groups than in HFC $(p=0.007)$ and HFR $(p=0.017)$, while HFC and HFR groups were not different $(p=0.946)$. After switching diets, mean triglyceride levels $(\mathrm{mg} / \mathrm{dL})$ prior to the diet switch were as follows: HFC NW 121.1 \pm 17.9 , HFC W $122.7 \pm 13.8$, HFR NW $68.8 \pm 8.2$, HFR W $65.7 \pm 6.6$, RC NW 78.0 \pm 9.9, RC W 62.3 \pm 6.5 . HFR mice exhibited similar triglyceride levels to $\mathrm{RC}(p=0.954)$ and lower levels compared to HFC $(p<0.001)$. Wheel-running did not affect triglycerides before $(p=0.362)$ or after food switch $(p=0.940)$.

\section{Liver Histology}

Before the food switch, mean liver lipodosis rankings were: HFC NW 1.0 \pm 0 , HFC W $1.0 \pm 0$, HFR NW $0.67 \pm 0.50$, HFR W $0.67 \pm 0.33$, RC NW $0.0 \pm 0.0$, RC W $0.25 \pm 0.25$. HFC mice exhibited increased hepatic lipidosis $(p=0.030)$, but HFR mice only had a nonsignificant increase in rankings $(p=0.088)$ compared to RC. No differences were present between HFR and HFC groups $(p=0.430)$. After the switch, mean liver lipodosis rankings were: HFC NW $1.29 \pm 0.19$, HFC W $1.22 \pm 0.22$, HFR NW $0.0 \pm 0.0$, HFR W $0.10 \pm 0.10$, RC
NW $0.17 \pm 0.17$, RC W $0.0 \pm 0.0$. HFC groups had fattier livers than both HFR $(p<0.001)$ and $\mathrm{RC}(p<0.001)$, while no difference was found between RC and HFR ( $p=0.97)$. Thus, mice continuously eating a high-fat diet (HFC) exhibited a continued increase of lipidosis, which was not observed in the other groups (HFR and $\mathrm{RC}$ ). Wheel-running produced no differences in level of hepatic lipidosis before $(p=0.520)$ or after switching diets $(p=0.427)$ (Fig. 5a-e).

\section{DISCUSSION}

These results illustrate that switching from highfat diets to regular rodent chow can produce rapid improvements of obesity (body mass and hepatic lipidosis) and T2DM-like characteristics (insulin, glucose tolerance) in a diet-induced obesity (DIO) mouse model. While most DIO models consume additional kcals on a high-fat diet, ${ }^{3,20}$ the mice in the current study and one other did not, ${ }^{24}$ yet were still obese compared to the controls. These results indicate that it is not just the increase in calories consumed that can lead to obesity in these DIO mice but food composition as well. Interestingly, both food switch (i.e., ad libitum feeding of regular chow) $)^{3,19}$ and caloric restriction of a high-fat diet ${ }^{19,25}$ produce significant improvements in obesity and insulin resistance in rodent models. While there are numerous parallel results between calorie restriction and switching to a low-fat diet, one remaining question is whether leptin levels are similarly affected by these two treatments. In the current study, switching to regular chow seems to completely reverse hyperleptinemia in mouse models, but mice with calorie restriction on a high-fat diet still had slightly elevated levels compared to controls, ${ }^{25}$ indicating that there may be hunger effects of restricted high-fat diet consumption compared to ad libitum regular chow treatment. A study which investigated both restricted high-fat diet and switch to regular chow showed mice having similarly reduced levels of leptin compared to high-fat diet consuming mice. ${ }^{19}$ Additional studies may be needed to fully determine whether it is the reductions in caloric intake or in fat content that most effectively reduce leptin levels in obese individuals.

In B6 mice, switching to regular chow can produce a complete reversal of obesity, as seen in the cur- 

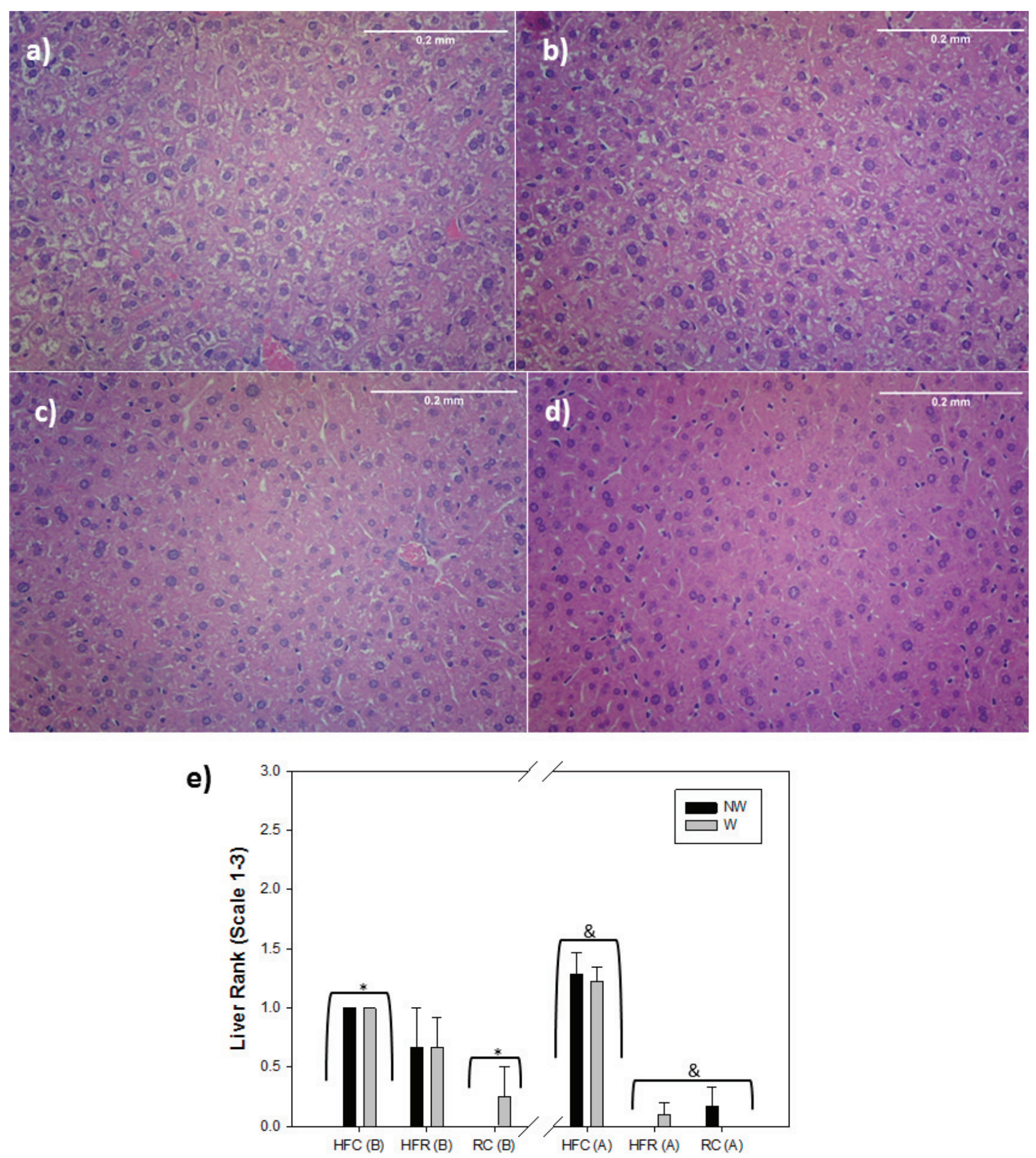

Figure 5. Examples of Liver Histology. a) Rank 3, b) Rank 2, c) Rank 1, d) Rank 0, e) Graphical representation of summarized liver rankings. Differences between the ranks were determined by the amount of lipid vacuolation (clear/white spaces) present in hepatocytes. ${ }^{*}=$ significantly different from each other, and ${ }^{\wedge}=$ HFC significantly greater than HFR and RC at $p<0.05$. The left side of the graph shows Before (indicated by B) and the right side shows After food switch (indicated by A).

rent study and one other, ${ }^{3}$ though other studies have found that the obesogenic effects of a high-fat diet are more persistent. ${ }^{20,26}$ These differences may be due to the duration of the high-fat diet feeding. Rodents 
fed a high-fat diet for shorter durations $(<17$ weeks, as was the case in this study) are able to have their obesity completely reversed by the switch to regular chow, ${ }^{3}$ but if further extended, permanent alterations in obesity and T2DM symptoms remain. ${ }^{26}$ On the other hand, Guo et $\mathrm{al}^{20}$ demonstrated that mice fed a high-fat diet for only 7 weeks do not fully recover from high-fat diet treatment and remained heavier than the controls throughout the study. The cause of this difference might be the age of the animals. While the current study and Parekh et $\mathrm{al}^{3}$ used young mice (4-6 weeks), Guo et al ${ }^{20}$ used relatively older mice (3 months); older animals ${ }^{27}$ and humans ${ }^{28}$ exhibit greater difficulty in losing body mass compared to younger individuals even with calorie restriction.

While the switch to regular chow led to the complete reversal of the obesogenic effects of high-fat diet HFR mice, the exercise produced no further improvements in body weight and T2DM symptoms. In a human study investigating the effects of simultaneous caloric restriction and exercise, combined exercise and calorie restriction produced no additional improvements in obesity markers, including body weight, compared to individuals with calorie restriction alone. ${ }^{29}$ This result suggests that as body mass decreases, further weight loss due to exercise becomes more difficult. Indeed, a human study has shown that exercise does not produce further significant weight loss in already lean persons, probably due to reduced energy expenditure in these individuals. ${ }^{30}$

The current study and other reports illustrate that exercise alone can produce minimal, though statistically significant, improvements in some T2DM symptoms in rodents fed a high-fat diet. ${ }^{15,24}$ Wheelrunning can also alleviate T2DM symptoms in mice selectively-bred ${ }^{31}$ or genetically altered ${ }^{32}$ for T2DM. However, the effects of exercise on obesity may be few at best, as multiple studies report only small improvements in the animal and human literature. In the current study, HFC W mice still exhibited similar levels of lipidosis and triglycerides compared to HFC NW and were increased compared to regular chow consuming mice. The reduced body mass seen in HFC W was small and non-lasting, implying that voluntary exercise had little-to-no effect on obesity characteristics. In contrast, exercise appears to be more beneficial in improving T2DM and metabolic disorder symptoms such as insulin resistance, ${ }^{15}$ which outcomes are verified by human studies, ${ }^{33,34}$ even without significant weight loss. On the other hand, this study and one other ${ }^{24}$ indicated that exercise had no effect on glucose tolerance, especially as time progressed, which may be surprising given that insulin levels are affected. The positive effects of exercise on insulin secretion might be due to its direct effect on the insulin release pathways and its conversion into active forms. Type- 2 diabetics are known to have increased inactive insulin and proinsulin, ${ }^{35}$ while wheel-running produces reductions in pancreatic proinsulin mRNA in rats. ${ }^{36}$ This reduction in proinsulin secretion might be the mechanism behind the reduced hyperinsulinemia seen in exercising individuals as insulin levels drop, whereas c-peptide levels remain similar between sedentary and exercising animals ${ }^{24}$ and humans. ${ }^{37}$ The unchanged levels of c-peptide or active insulin could be the reason why overall insulin levels are affected by exercise, but not glucose tolerance.

The current study and others ${ }^{15,24}$ show that voluntary wheel-running and forced exercise ${ }^{17,18}$ via a treadmill or motorized running-wheel demonstrate moderate improvements comparable to each other regarding obesity and T2DM symptoms in exercising rodents consuming a high-fat diet. One study using rats examining both forced and voluntary exercise simultaneously illustrated that regardless of exercise method, exercise produces improvements in insulin resistance. ${ }^{38}$ Despite these parallel results linking improvements in T2DM and obesity symptoms to any form of exercise, there are differences between forced and voluntary exercise regarding other physiological outcomes. For example, mice consuming a high-fat diet have shown reduced inflammation when given access to running-wheels; ${ }^{15}$ conversely, forced exercise may promote inflammation ${ }^{39}$ and differently affect the gut microbiome compared to voluntary wheel-running in rodent models. ${ }^{40}$ Future studies investigating the effects of exercise on physiology will need to establish which parameters they wish to investigate and whether to use voluntary or forced exercise in the experiments.

The current results corroborate a previous study revealing that mice consuming a high-fat diet give evidence of reduced activity as time progresses. ${ }^{41}$ 
This reduction in activity manifests itself by reductions in activity during the active period (dark) and with reductions in the number of counts per bout and length of bouts, but not number of bouts per day. These results mean that mice continuously fed a high-fat diet have reduced output during locomotor events compared to controls, but have the same number of locomotor events (i.e., they move the same number of times per day but make fewer movements during those times). Surprisingly, the current results and a previous study ${ }^{24}$ demonstrate that when given running-wheel access, mice continuously consuming a high-fat diet do not show the same reduction in locomotor activity as their sedentary counterparts. This result indicates that the increased sedentary behavior in obese mice consuming a high-fat diet without a running-wheel might not be due to their inability to move due to increased body mass. Voluntary wheelrunning is a rewarding activity for rodents ${ }^{42}$ and leads to increased activity compared to animals without running-wheels. ${ }^{21}$ In humans, obesity can lead to avoidance of physical activity. ${ }^{43,44}$ Physical inactivity and obesity can be locked into a vicious cycle where obesity can make individuals more sedentary, while inactivity can be more likely to make individuals more obese and unhealthy. ${ }^{45}$ These results indicate that individuals with obesity must make it a priority to maintain an exercise regimen to promote healthy outcomes, prevent the development of T2DM, and thwart the positive feedback of increased obesity and inactivity feeding into each other.

In conclusion, removing a high-fat diet and replacing it with regular chow was effective in quickly reversing the increased obesity and T2DM characteristics in mice previously consuming a high-fat diet. Voluntary wheel-running did not produce further improvements in these characteristics, as mice with the high-fat diet removed became as lean as control mice even without running-wheels. In contrast, wheelrunning by itself produced improvements in insulin levels in mice consuming a high-fat diet. Nevertheless, these mice still exhibited obesity, hepatic lipidosis, and other increased diabetic-like symptoms (glucose tolerance) compared to mice eating regular chow, indicating that exercise alone cannot completely prevent the negative health consequences of an unhealthy lifestyle.

\section{ACKNOWLEDGEMENTS}

This work was supported by Bridgewater State University ATP and CARS programs. Madison Chasse and Alexis Cushman facilitated in data collection. We thank Dana-Farber/Harvard Cancer Center in Boston, MA, for the use of the Rodent Histopathology Core, which provided histological service. Dana-Farber/ Harvard Cancer Center is supported in part by a NCI Cancer Center Support Grant \# NIH 5 P30 CA06516.

\section{CONFLICT OF INTEREST}

The authors declare that they have no conflict of interest.

\section{REFERENCES}

1. Kahn SE, Hull RL, Utzschneider KM, 2006 Mechanisms linking obesity to insulin resistance and type 2 diabetes. Nature 444: 840-846.

2. Enriori PJ, Evans AE, Sinnayah P, Cowley MA, 2006 Leptin resistance and obesity. Obesity (Silver Spring) 14: Suppl 5: 254-258.

3. Parekh PI, Petro AE, Tiller JM, Feinglos MN, Surwit RS, 1998 Reversal of diet-induced obesity and diabetes in C57BL/6J mice. Metabolism 47: 1089-1096.

4. Redman LM, Ravussin E, 2011 Caloric restriction in humans: impact on physiological, psychological, and behavioral outcomes. Antioxid Redox Signal 14: 275-287.

5. Johnson ML, Distelmaier K, Lanza IR, et al, 2016 Mechanism by Which Caloric Restriction Improves Insulin Sensitivity in Sedentary Obese Adults. Diabetes 65: 74-84.

6. Kim H, Kang H, Heo RW, et al, 2016 Caloric restriction improves diabetes-induced cognitive deficits by attenuating neurogranin-associated calcium signaling in high-fat diet-fed mice. J Cereb Blood Flow Metab 36: 1098-1110.

7. Wang X, Cheng M, Zhao M, et al, 2013 Differential effects of high-fat-diet rich in lard oil or soybean oil on osteopontin expression and inflammation of adipose tissue in diet-induced obese rats. Eur J Nutr 52: 1181-1189.

8. Roberts CK, 2014. Combined diet and exercise interventions can improve metabolic outcomes in obese children, even with only small changes in weight. Evid Based Med 19: e9.

9. Ross R, Janssen I, Dawson J, et al, 2004 Exercise-induced reduction in obesity and insulin resistance in women: a randomized controlled trial. Obes Res 12: 789-798.

10. Richardson CR, Newton TL, Abraham JJ, Sen A, Jimbo M, Swartz AM, 2008 A meta-analysis of pedometerbased walking interventions and weight loss. Ann Fam 
Med 6: 69-77.

11. Luke A, Cooper RS, 2013 Physical activity does not influence obesity risk: time to clarify the public health message. Int J Epidemiol 42: 1831-1836.

12. Thomas DM, Bouchard C, Church T, et al, Why do individuals not lose more weight from an exercise intervention at a defined dose? An energy balance analysis. 2013 Obes Rev 13: 835-847.

13. Lafortune G, De Looper M, Balestat G, Health at a glance 2009: OECD indicators. 2009 OECD Publishing, Paris

14. Kim Y, Park H, 2013 Does Regular Exercise without Weight Loss Reduce Insulin Resistance in Children and Adolescents? Int J Endocrinol 2013: 402592.

15. Bradley RL, Jeon JY, Liu FF, Maratos-Flier E, 2008 Voluntary exercise improves insulin sensitivity and adipose tissue inflammation in diet-induced obese mice. Am J Physiol Endocrinol Metab 295: E586-594.

16. Carter LG, Ngo Tenlep SY, Woollett LA, Pearson KJ, 2015 Exercise Improves Glucose Disposal and Insulin Signaling in Pregnant Mice Fed a High Fat Diet. J Diabetes Metab 6: 634.

17. Vieira VJ, Valentine RJ, Wilund KR, Woods JA, 2009 Effects of diet and exercise on metabolic disturbances in high-fat diet-fed mice. Cytokine 46: 339-345.

18. Kang SS, Jeraldo PR, Kurti A, et al, 2014 Diet and exercise orthogonally alter the gut microbiome and reveal independent associations with anxiety and cognition. Mol Neurodegener 9: 36.

19. Hoevenaars FP, Keijer J, Herreman L, et al, 2014 Adipose tissue metabolism and inflammation are differently affected by weight loss in obese mice due to either a high-fat diet restriction or change to a low-fat diet. Genes Nutr 9: 391.

20. Guo J, Jou W, Gavrilova O, Hall KD, 2009 Persistent diet-induced obesity in male C57BL/6 mice resulting from temporary obesigenic diets. PLoS One 4: e5370.

21. Nascimento NF, Hicks JA, Carlson KN, Hatzidis A, Amaral DN, Seggio JA, 2016 6-h advances alter circadian activity patterns, fasting glucose, and insulin levels in C57BL6/J mice. Biological Rhythm Research 47: 133-143.

22. Ahmad ST, Steinmetz SB, Bussey HM, Possidente B, Seggio JA, 2013 Larval ethanol exposure alters freerunning circadian rhythm and per Locus transcription in adult D. melanogaster period mutants. Behav Brain Res 241: 50-55.

23. Hubscher SB, Burt AD, Portmann BC, Ferrell LD, 2011 MacSween's Pathology of the Liver. Churchill Livingstone, London.

24. Hicks JA, Hatzidis A, Arruda NL, et al, 2016 Voluntary wheel-running attenuates insulin and weight gain and affects anxiety-like behaviors in C57BL6/J mice exposed to a high-fat diet. Behav Brain Res 310: 1-10.

25. Park S, Park NY, Valacchi G, Lim Y, 2012 Calorie restriction with a high-fat diet effectively attenuated inflammatory response and oxidative stress-related markers in obese tissues of the high diet fed rats. Mediators Inflamm 2012: 984643.

26. Hill JO, Dorton J, Sykes MN, Digirolamo M, 1989 Reversal of dietary obesity is influenced by its duration and severity. Int J Obes 13: 711-722.

27. Sugatani J, Sadamitsu S, Yamaguchi M, et al, 2014 Antiobese function of platelet-activating factor: increased adiposity in platelet-activating factor receptor-deficient mice with age. FASEB J 28: 440-452.

28. Villareal DT, Apovian CM, Kushner RF, et al, 2005 Obesity in older adults: technical review and position statement of the American Society for Nutrition and NAASO, The Obesity Society. Obes Res 13: 1849-1863.

29. Larson-Meyer DE, Heilbronn LK, Redman LM, et al, 2006 Effect of calorie restriction with or without exercise on insulin sensitivity, beta-cell function, fat cell size, and ectopic lipid in overweight subjects. Diabetes Care 29: 1337-1344.

30. Bonomi AG, Soenen S, Goris AH, Westerterp KR, 2013 Weight-loss induced changes in physical activity and activity energy expenditure in overweight and obese subjects before and after energy restriction. PLoS One 8: e59641.

31. Nascimento NF, Hicks JA, Carlson KN, et al, 2016 Long-term wheel-running and acute 6 -h advances alter glucose tolerance and insulin levels in TALLYHO/JngJ mice. Chronobiol Int 33: 108-116.

32. Irani BG, Xiang Z, Moore MC, Mandel RJ, HaskellLuevano C, 2005 Voluntary exercise delays monogenetic obesity and overcomes reproductive dysfunction of the melanocortin-4 receptor knockout mouse. Biochem Biophys Res Commun 326: 638-644.

33. Duncan GE, Perri MG, Theriaque DW, Hutson AD, Eckel RH, Stacpoole PW, 2003 Exercise training, without weight loss, increases insulin sensitivity and postheparin plasma lipase activity in previously sedentary adults. Diabetes Care 26: 557-562.

34. Fedewa MV, Gist NH, Evans EM, Dishman RK, 2014 Exercise and insulin resistance in youth: a meta-analysis. Pediatrics 133: e163-174.

35. Ward WK, LaCava EC, Paquette TL, Beard JC, Wallum BJ, Porte D, 1987 Disproportionate elevation of immunoreactive proinsulin in type 2 (non-insulin-dependent) diabetes mellitus and in experimental insulin resistance. Diabetologia 30: 698-702.

36. Koranyi LI, Bourey RE, Slentz CA, Holloszy JO, Permutt MA, 1991 Coordinate reduction of rat pancreatic islet glucokinase and proinsulin mRNA by exercise training. Diabetes 40: 401-404.

37. Duvivier BM, Schaper NC, Bremers MA, et al, 2013 Minimal intensity physical activity (standing and walking) of longer duration improves insulin action and plasma lipids more than shorter periods of moderate to vigorous exercise (cycling) in sedentary subjects when energy expenditure is comparable. PLoS One 8: e55542.

38. Boersma GJ, Barf RP, Benthem L, van Dijk G, Scheurink 
AJ, 2012 Forced and voluntary exercise counteract insulin resistance in rats: the role of coping style. Horm Behav 62: 93-98.

39. Cook MD, Martin SA, Williams C, et al, 2013 Forced treadmill exercise training exacerbates inflammation and causes mortality while voluntary wheel training is protective in a mouse model of colitis. Brain Behav Immun 33: 46-56.

40. Allen JM, Berg Miller ME, Pence BD, et al, 2015 Voluntary and forced exercise differentially alters the gut microbiome in C57BL/6J mice. J Appl Physiol 118: 1059-1066.

41. Kohsaka A, Laposky AD, Ramsey KM, et al, 2007 High-fat diet disrupts behavioral and molecular circadian rhythms in mice. Cell Metab 6: 414-421.

42. Greenwood BN, Foley TE, Le TV, et al, 2011 Long-term voluntary wheel running is rewarding and produces plasticity in the mesolimbic reward pathway. Behav Brain Res 217: 354-362.

43. Bauman AE, Reis RS, Sallis JF, et al, 2012 Correlates of physical activity: why are some people physically active and others not? Lancet 380: 258-271.

44. Vartanian LR, Herman CP, Polivy J, 2008 Judgments of body weight based on food intake: a pervasive cognitive bias among restrained eaters. Int J Eat Disord 41: 64-71.

45. Pietiläinen KH, Kaprio J, Borg P, et al, 2008 Physical inactivity and obesity: a vicious circle. Obesity (Silver Spring) 16: 409-414. 